\title{
A high conversion-gain Q-band InP DHBT subharmonic mixer using LO frequency doubler
}

Johansen, Tom Keinicke; Vidkjær, Jens; Krozer, Viktor; Konczykowska, A.; Riet, M.; Jorge, F.; Djurhuus, Torsten

Published in:

IEEE Transactions on Microwave Theory and Techniques

Link to article, DOI:

10.1109/TMTT.2008.916892

Publication date:

2008

Document Version

Publisher's PDF, also known as Version of record

Link back to DTU Orbit

Citation (APA):

Johansen, T. K., Vidkjær, J., Krozer, V., Konczykowska, A., Riet, M., Jorge, F., \& Djurhuus, T. (2008). A high conversion-gain Q-band InP DHBT subharmonic mixer using LO frequency doubler. IEEE Transactions on Microwave Theory and Techniques, 56(3), 613-619. https://doi.org/10.1109/TMTT.2008.916892

\section{General rights}

Copyright and moral rights for the publications made accessible in the public portal are retained by the authors and/or other copyright owners and it is a condition of accessing publications that users recognise and abide by the legal requirements associated with these rights.

- Users may download and print one copy of any publication from the public portal for the purpose of private study or research.

- You may not further distribute the material or use it for any profit-making activity or commercial gain

- You may freely distribute the URL identifying the publication in the public portal 


\title{
A High Conversion-Gain $Q$-Band InP DHBT Subharmonic Mixer Using LO Frequency Doubler
}

\author{
Tom K. Johansen, Member, IEEE, Jens Vidkjær, Member, IEEE, Viktor Krozer, Senior Member, IEEE, \\ Agnieszka Konczykowska, Senior Member, IEEE, Muriel Riet, Filipe Jorge, and Torsten Djurhuus
}

\begin{abstract}
The paper presents analysis and design of a $Q$-band subharmonic mixer (SHM) with high conversion gain. The SHM consists of a local oscillator (LO) frequency doubler, RF pre-amplifier, and single-ended mixer. The SHM has been fabricated in a high-speed InP double heterojunction bipolar transistor (DHBT) technology using coplanar waveguide structures. To the best of our knowledge, this is the first demonstration of an SHM using InP DHBT technology at millimeter-wave frequencies. The measured results demonstrate a conversion gain of $10.3 \mathrm{~dB}$ at $45 \mathrm{GHz}$ with an LO power of only $1 \mathrm{~mW}$. The fundamental mixing product is suppressed by more than $24 \mathrm{~dB}$ and the output $P_{1 \mathrm{~dB}}$ is around $-6 \mathrm{dBm}$. The mixer is broadband with a conversion gain above $7 \mathrm{~dB}$ from 40 to $50 \mathrm{GHz}$. The conversion gain for the fabricated SHM is believed to be among the best ever reported for millimeterwave SHMs.
\end{abstract}

Index Terms-Coplanar waveguides (CPWs), heterojunction bipolar transistors (HBTs), millimeter-wave circuit, mixer analysis, monolithic microwave integrated circuits (MMICs), nonlinear circuit analysis, subharmonic mixer (SHM).

\section{INTRODUCTION}

$\mathbf{S}$ UBHARMONIC mixers (SHMs) have long been recognized as an interesting alternative to fundamental frequency mixers in millimeter-wave systems. The reason being that SHMs require only half the local oscillator (LO) frequency, resulting in an easier design of high spectral purity LOs and relaxation of the power requirements. In addition to this, SHMs offer inherent RF/LO isolation, which is important in receiver applications where unwanted radiation of $\mathrm{LO}$ power from the RF port must be minimized.

The most common SHM topology consists of a pair of antiparallel Schottky barrier diodes [1], [2]. However, this topology suffers from significant conversion loss and requires additional amplification. Furthermore, high cutoff frequency Schottky barrier diodes are generally not available in monolithic microwave integrated circuit (MMIC) technologies. Another widely used SHM topology extends the standard Gilbert cell mixer with a stacked LO switching core [3]. At millimeter-wave frequencies, the LO transistors do not operate well as switches,

Manuscript received November 27, 2006; revised September 26, 2007. This work was supported in part by the European Union under Project GIBON.

T. K. Johansen, J. Vidkjær, V. Krozer, and T. Djurhuus are with the Electro-Science Section, Ørsted • DTU Department, Technical University of Denmark, DK-2800 Kgs. Lyngby, Denmark (e-mail: tkj@oersted.dtu.dk; jv@oersted.dtu.dk; vk@oersted.dtu.dk; tdj@oersted.dtu.dk).

A. Konczykowska, M. Riet, and F. Jorge are with the Alcatel-Thales III-V Laboratory, F-91461 Marcoussis, France (e-mail: agnieszka.konczykowska@35lab.fr; muriel.riet@3-5lab.fr; filipe.jorge@3-5lab.fr).

Digital Object Identifier 10.1109/TMTT.2008.916892 resulting in poor conversion gain and suppression of the fundamental mixing product. Furthermore, this topology requires a rather large supply voltage (typically around $7 \mathrm{~V}$ ). Recently, a balanced SHM topology was reported [4], which provides excellent isolation between the LO and RF ports. This topology, however, provides no suppression of the fundamental mixing product.

This paper reports on a high-gain InP double heterojunction bipolar transistor (DHBT) SHM for $Q$-band applications. The SHM topology consists of an LO frequency doubler, RF preamplifier, and single-ended mixer, as previously proposed for pHEMT-based SHMs [5]. The use of an LO frequency doubler in front of a mixing stage in order to obtain the wanted subharmonic mixing functionality has also recently been demonstrated in 0.18- $\mu \mathrm{m}$ CMOS technology [6].

The design of SHMs is complicated due to the consecutive nonlinear processes of frequency doubling and frequency mixing in one circuit. At $Q$-band frequencies and above, accurate passive circuit design further complicates the design procedure. Here we present a partly analytical approach for the design of an SHM, which greatly facilitates the choice of the LO power, transistor bias points, and embedding impedances. The passive structures are designed using extensive electromagnetic (EM) simulations.

The measured results on the fabricated InP DHBT SHM demonstrate high conversion gain at very low LO power and good suppression of the fundamental mixing product.

\section{Design TheORY}

To illustrate the mixing principle used in the SHM topology, we consider the circuit shown in Fig. 1(a). The applied LO signal is a large second harmonic signal $v_{\mathrm{LO}}(t)=V_{\mathrm{LO}} \cos \left(2 \omega_{\mathrm{LO}} t\right)$ generated by a preceding frequency doubler transistor device. The large LO signal gives rise to a time-varying circuit. An approximate analysis of the single-ended mixer is possible by considering the simplified time-varying small-signal equivalent circuit model shown in Fig. 1(b) [7]. In this model, only the transconductance $g_{m}(t)$ is assumed to be time varying, while the base-emitter capacitance $C_{\text {be }}$ is represented by its time-averaged value. The resistances $R_{b}$ and $R_{e}$ in the input loop of the HBT are lumped into a single resistor. This representation provides a good tradeoff between analysis accuracy and interpretation of the final results for conversion gain and input impedance. The exponential nonlinear $I / V$ characteristic of an HBT device leads to a transconductance waveform given as

$g_{m}(t)=G_{0}+2 G_{1} \cos \left(2 \omega_{\mathrm{LO}} t+\phi_{1}\right)+2 G_{2} \cos \left(4 \omega_{\mathrm{LO}} t+\phi_{2}\right)+\cdots$ 


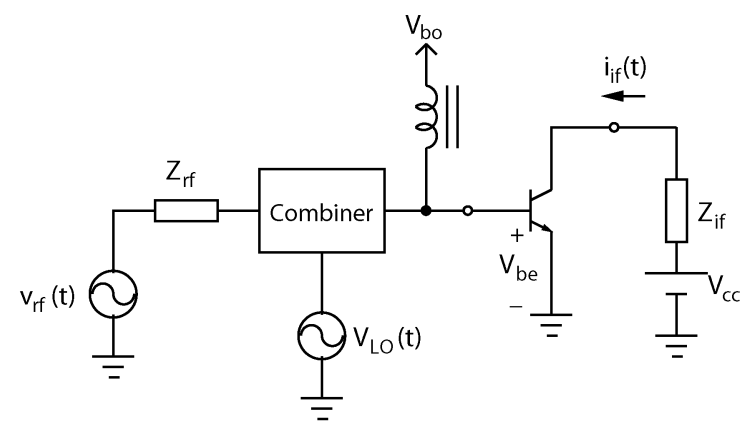

(a)
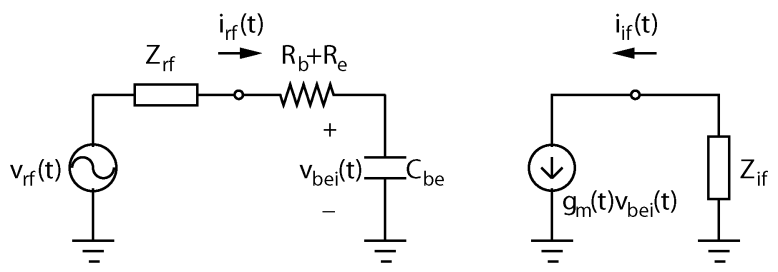

(b)

Fig. 1. (a) Single-ended transconductance mixer principle. (b) Simplified timevarying small-signal equivalent circuit.

where $G_{n} \angle \phi_{n}$ represent complex Fourier coefficients, dependent on the LO drive level $V_{\mathrm{LO}}$, and the base bias voltage $V_{b o}$. As was discussed in [8], the drive voltage across the internal base-emitter junction $V_{\mathrm{be}} i(t)$ in HBTs cannot, in general, be assumed sinusoidal and, therefore, it is not valid to express the Fourier coefficients in terms of modified Bessel functions, as is commonly employed in mixer analysis [9]. The time-varying transconductance give rise to mixing frequencies at

$$
\omega_{n}=\omega_{\text {if }}+n 2 \omega_{\mathrm{LO}}, \quad n=0, \pm 1, \pm 2, \pm 3, \ldots
$$

where $\omega_{\text {if }}=\left|2 \omega_{\text {LO }}-\omega_{\text {rf }}\right|$ is the IF angular frequency [1]. The base-emitter capacitance consists of the sum of the time-varying depletion $C_{d}(t)$ and diffusion capacitances $g_{m}(t) \tau_{f}$ given as

$$
C_{\mathrm{be}}(t)=\left.\frac{d Q_{\mathrm{be}}}{d V_{\mathrm{be}}}\right|_{V_{\mathrm{be}} i(t)}=C_{d}(t)+g_{m}(t) \tau_{f}
$$

where $Q_{\mathrm{be}}$ is the base-emitter junction charge and $\tau_{f}$ is the forward transit time of the HBT devices. Despite its time-varying nature, it is assumed that the base-emitter capacitance contribute little to the frequency conversion in the single-ended mixer and a time-averaged value

$$
C_{\mathrm{be}}=\frac{2}{T_{\mathrm{LO}}} \int_{0}^{T_{\mathrm{LO}} / 2} C_{\mathrm{be}}(t) d t=C_{d 0}+G_{0} \tau_{f}
$$

where $T_{\mathrm{LO}}=2 \pi / \omega_{\mathrm{LO}}$ is the period of the LO signal and $C_{d 0}$, the average value of the depletion capacitance, can be used in the time-varying small-signal equivalent circuit.

The input impedance can be determined from traditional linear time-invariant analysis and is given as

$$
Z_{\text {in }}=R_{b}+R_{e}+\frac{1}{j \omega_{\mathrm{rf}}\left(C_{d 0}+G_{0} \tau_{f}\right)}
$$

TABLE I

ElECTRICAL PARAMETERS FOR THE NONLINEAR InP DHBT MODEL $\left(A_{e}=10 \times 2.0 \mu \mathrm{m}^{2}\right)$

\begin{tabular}{|c|c|c|c|}
\hline Parameter & Value & Parameter & Value \\
\hline$I_{s}[\mathrm{fA}]$ & 4.5 & $R_{c}[\Omega]$ & 9.9 \\
\hline$N_{f}$ & 1.13 & $\tau_{f}[p S]$ & 0.53 \\
\hline$\beta_{f}$ & 28.1 & $C_{j e}[\mathrm{fF}]$ & 24.6 \\
\hline$R_{b}[\Omega]$ & 23.0 & $V_{j e}[\mathrm{fF}]$ & 0.709 \\
\hline$R_{e}[\Omega]$ & 3.0 & $M_{j e}$ & 0.34 \\
\hline
\end{tabular}

with a constant real part, but with a base bias and LO drive level dependent imaginary part.

To analyze the conversion gain of a time-varying circuit, conversion matrices are introduced. The conversion matrix form of the time-varying transconductance can be written as

$$
\left[\begin{array}{c}
I_{\mathrm{if},-1}^{*} \\
I_{\mathrm{if}, 0} \\
I_{\mathrm{if}, 1}
\end{array}\right]=\left[\begin{array}{ccc}
G_{0} & G_{1} \angle \phi_{1}^{*} & G_{2} \angle \phi_{2}^{*} \\
G_{1} \angle \phi_{1} & G_{0} & G_{1} \angle \phi_{1}^{*} \\
G_{2} \angle \phi_{2} & G_{1} \angle \phi_{1} & G_{0}
\end{array}\right]\left[\begin{array}{c}
V_{\mathrm{be},-1}^{*} \\
V_{\mathrm{be}, 0} \\
V_{\mathrm{be} i, 1}
\end{array}\right]
$$

where $I_{\text {if }, n}$ are the resulting currents at the mixing frequency $\omega_{n}$ for a voltage $V_{\mathrm{be} i, m}$ at the relevant excitation frequency $\omega_{m}$, and the asterisks denote a complex conjugate. The wanted IF frequency current component is $I_{\text {if }, 0}$ and the excitation voltage component at the RF frequency is $V_{\text {bei,1. }}$. For the simplified time-varying equivalent circuit model in Fig. 1(b) feedback is absent and, therefore, the relation between the output current at the IF frequency and the internal base-emitter voltage at the RF frequency reduces to

$$
I_{\text {if }, 0}=G_{1} \angle \phi_{1}^{*} \cdot V_{\mathrm{be} i, 1} \cdot
$$

Currents at all other mixing products are assumed to be short circuited to ground through the embedded impedance $Z_{\text {if }}$. Relating the intrinsic base-emitter voltage to the external RF excitation $v_{\mathrm{rf}}=V_{\mathrm{RF}} \cos \left(\omega_{\mathrm{rf}} t\right)$ gives

$$
I_{\mathrm{if}, 0}=\frac{G_{1} \angle \phi_{1}^{*}}{1+j \omega_{\mathrm{rf}}\left(Z_{\mathrm{rf}}+R_{b}+R_{e}\right)\left(C_{d 0}+G_{0} \tau_{f}\right)} \frac{V_{\mathrm{RF}}}{2}
$$

where the factor of 2 comes from the fact that $I_{\text {if }, 0}$ represents a positive frequency phasor. The final expression for the transducer power conversion gain becomes

$$
G_{c}=\frac{G_{1}^{2}}{\omega_{\mathrm{rf}}^{2}\left(R_{b}+R_{e}\right)\left(C_{d 0}+G_{0} \tau_{f}\right)^{2}} \Re\left(Z_{\text {if }}\right)
$$

where $Z_{\text {rf }}=Z_{\text {in }}^{*}$ have been assumed and $\Re\left(Z_{\text {if }}\right)$ represents the real part of $Z_{\text {if }}$.

\section{SHM DESIGN}

It will now be explained how the single-ended mixer theory described in Section III can be used in the design of the SHM. The electrical parameters of the nonlinear model for the InP DHBTs used for the initial design considerations are given in Table I. This model neglects the internal feedback through the base-collector capacitance and the strong bias dependence of the forward transit time experienced in InP DHBTs. For the final design verification, the more accurate Agilent HBT large-signal model is used. As was previously reported by the authors in [10], 


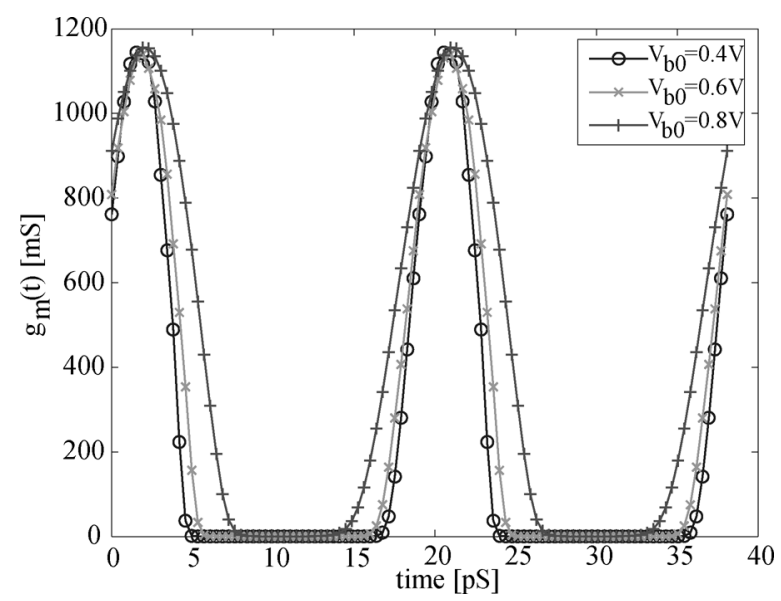

Fig. 2. Calculated time-varying transconductance waveform for various base bias voltages. The $\mathrm{LO}$ excitation is at $2 f_{\mathrm{LO}}=52.5 \mathrm{GHz}$.

this large-signal model accurately predicts the performance of InP DHBTs at millimeter-wave frequencies.

An important issue in the design of the SHM is to find the necessary LO drive voltage for the single-ended mixer, as this will have influence on the design of the frequency doubler preceding the single-ended mixer. The $\mathrm{LO}$ drive voltage $V_{\mathrm{LO}}$ determines the peak current in the InP DHBT device together with the base bias voltage $V_{b 0}$. The maximum current rating $I_{\max }$ of the $\mathrm{InP}$ DHBT device, therefore, leads to a constraint on the LO drive voltage and base bias voltage. To assure a maximum current of $I_{\max }=30 \mathrm{~mA}$ at an LO excitation of $2 f_{\mathrm{LO}}=52.5 \mathrm{GHz}$ even if the conduction angle changes, the following empirical relation is found from simulations:

$$
V_{\mathrm{LO}}=-1.1 V_{b 0}+1.1 .
$$

To find the LO drive voltage for optimum conversion gain from (9) under the constraint of (10), a single-tone harmonic balance simulation at $2 f_{\mathrm{LO}}=52.5 \mathrm{GHz}$ is first performed to determine the voltage waveform $V_{\mathrm{be}} i(t)$ across the intrinsic base-emitter junction of the InP DHBT device in the single-ended mixer. Once the voltage waveform is known, the time-varying transconductance and time-varying base-emitter depletion capacitance can be calculated.

The time-varying transconductance for different base bias voltages is shown in Fig. 2. It is observed that due to the constraint given by (10), the peak height is constant and only the conduction angle changes. The Fourier coefficients $G_{1} \angle \phi_{1}, G_{0}$, and $C_{d 0}$ are determined from the time-varying transconductance and time-varying base-emitter depletion capacitance and are needed in the calculation of the conversion gain using (9). In Fig. 3, the calculated conversion gain is shown versus LO drive voltage for an RF excitation at $f_{\mathrm{RF}}=50 \mathrm{GHz}$ and LO excitation at $2 f_{\mathrm{LO}}=52.5 \mathrm{GHz}$. To verify the assumptions employed in the single-ended transconductance mixer analysis, the result from a two-tone harmonic balance simulation performed with Agilent ADS $^{1}$ is also shown in Fig. 3. Even at millimeter-wave frequencies, the simple time-varying small-signal equivalent circuit in Fig. 1(b) is observed to give an acceptable estimate of the conversion gain with the InP DHBTs. Importantly,

${ }^{1}$ ADS 2004A, Agilent Technol Inc., Palo Alto, CA

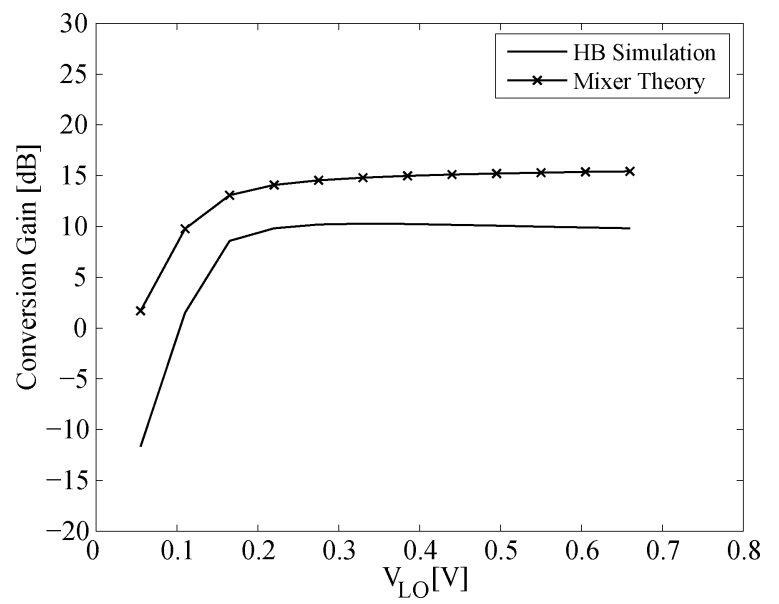

Fig. 3. Calculated (symbols) and simulated (line) conversion gain versus LO drive voltage. RF excitation at $f_{\mathrm{RF}}=50 \mathrm{GHz}$ and $\mathrm{LO}$ excitation at $2 f_{\mathrm{LO}}=$ $52.5 \mathrm{GHz}$.

the required minimum LO drive level is similar for both simulations and can, hence, be accurately determined from the analytical formula presented above. It should be mentioned that the difference between the calculated and simulated conversion gain comes from neglecting the internal feedback caused by $R_{e}$, the assumption of operation far above the $\beta$ cutoff frequency, and the use of a time-average base-emitter capacitance $C_{\mathrm{be}}$.

From Fig. 3, a saturation in conversion gain for LO drive voltages $>0.2 \mathrm{~V}$ is observed. This saturation can be explained from (9) considering that, for small conduction angles, the ratio $G_{1} / G_{0}$ is limited to a factor of approximately 2 . The conversion gain of the single-ended mixer stage is thus optimized if the frequency-doubler stage can deliver an LO drive voltage $>0.2 \mathrm{~V}$ at the base of the mixing InP DHBT device.

The SHM including the frequency doubler, RF pre-amplifier, and single-ended mixer is shown in Fig. 4. The single-ended mixer consists of the device $Q_{3}$, the $\lambda / 4 @ 2 f_{\text {LO }}$ line short-circuited with a capacitor for base bias injection, and the IF matching circuit formed by capacitor $C_{\mathrm{IF}}$ and inductor $L_{\mathrm{IF}}$. The IF matching circuit assures that unwanted mixing products at the output are shorted to ground, as was also assumed in the mixer analysis. The frequency doubler should convert the externally applied $\mathrm{LO}$ excitation at $f_{\mathrm{LO}}=26.25 \mathrm{GHz}$ into a $2 f_{\mathrm{LO}}=52.5 \mathrm{GHz}$ frequency signal with sufficient amplitude $\left(V_{\mathrm{LO}}>0.2 \mathrm{~V}\right)$ to drive the single-ended mixer. The frequency doubler design is based on reactive termination at the second harmonic at the input side of device $Q_{2}$ and short-circuit termination at the fundamental at the output side [11]. The second harmonic reactive termination implemented with the $\alpha \lambda @ f_{\mathrm{LO}}$ shorted line in Fig. 4 increases the conversion gain of the frequency doubler for a certain range of $\alpha$ values, as demonstrated in Fig. 5. This line is shorted by a capacitor to allow for the base bias injection needed for the device $Q_{2}$. The short-circuit termination at the fundamental frequency at the output assures stable operation [7] and is implemented with the $\lambda / 4 @ f_{\mathrm{LO}}$ open stub in Fig. 4 . The RF pre-amplifier is included to separate the output of the frequency doubler from the RF input and reduce the noise contribution from the single-ended mixer. The device $Q_{1}$ is biased for minimum noise at $f_{\mathrm{RF}}=50 \mathrm{GHz}$ through the $\lambda / 4 @ f_{\mathrm{RF}}$ shorted line. 


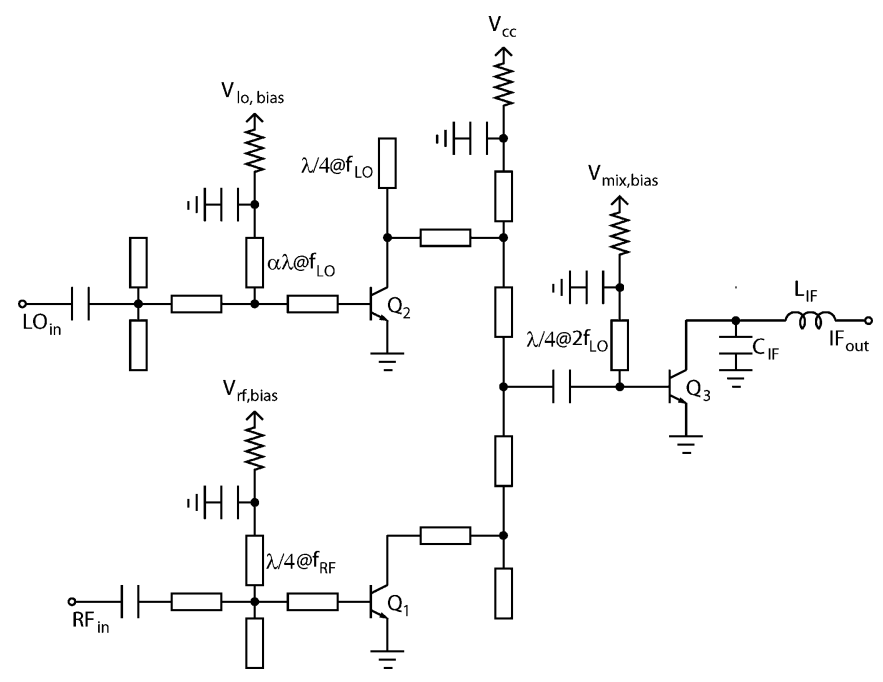

Fig. 4. Schematic of the SHM circuit.

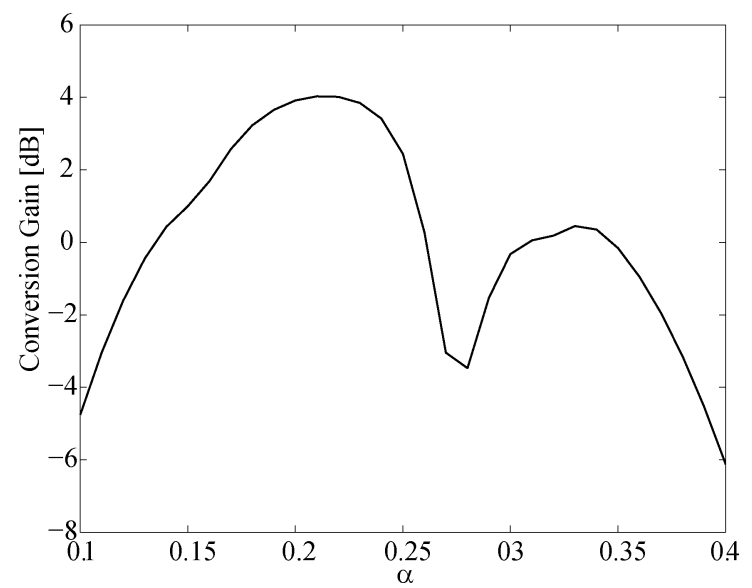

Fig. 5. Simulated frequency-doubler conversion gain versus line parameter $\alpha$. LO excitation at $f_{\mathrm{LO}}=26.25 \mathrm{GHz}$.

The matching circuit provides simultaneously power and noise match. The RF pre-amplifier stage adds a gain of $4.6 \mathrm{~dB}$ to the overall conversion gain of the SHM. The simulated conversion gain of the SHM is around $9.9 \mathrm{~dB}$ so the conversion gain of the single-ended mixer stage itself is expected to be around $5.3 \mathrm{~dB}$. A noise figure of $\mathrm{NF}=10.3 \mathrm{~dB}$ is found from simulations of the SHM using the $Y$-factor method. This is acceptable taking into account that the InP DHBT devices exhibits a rather high minimum noise figure of $\mathrm{NF}_{\min }=6.9 \mathrm{~dB}$ at $50 \mathrm{GHz}$.

\section{EXPERIMENTAL RESULTS AND DISCUSSION}

The SHM has been fabricated in a high-speed InP/InGaAs DHBT circuit technology developed at the Alcatel-Thales III-V Laboratory, Marcoussis, France. The transistors in this technology exhibit $180 / 210 \mathrm{GHz} f_{t} / f_{\max }$, breakdown voltage $\mathrm{BV}_{\text {ceo }}>7 \mathrm{~V}$, and yield better than 99\% [12]. The technology offers three $\mathrm{Au} / \mathrm{Ti}$ metallization layers, Ti resistors, and $\mathrm{SiN}$ metal-insulator-metal (MIM) capacitors. The microphotograph of the fabricated SHM is shown in Fig. 6. The transmission lines are implemented as coplanar waveguide (CPW) structures. CPW discontinuities and spiral inductor were modeled using

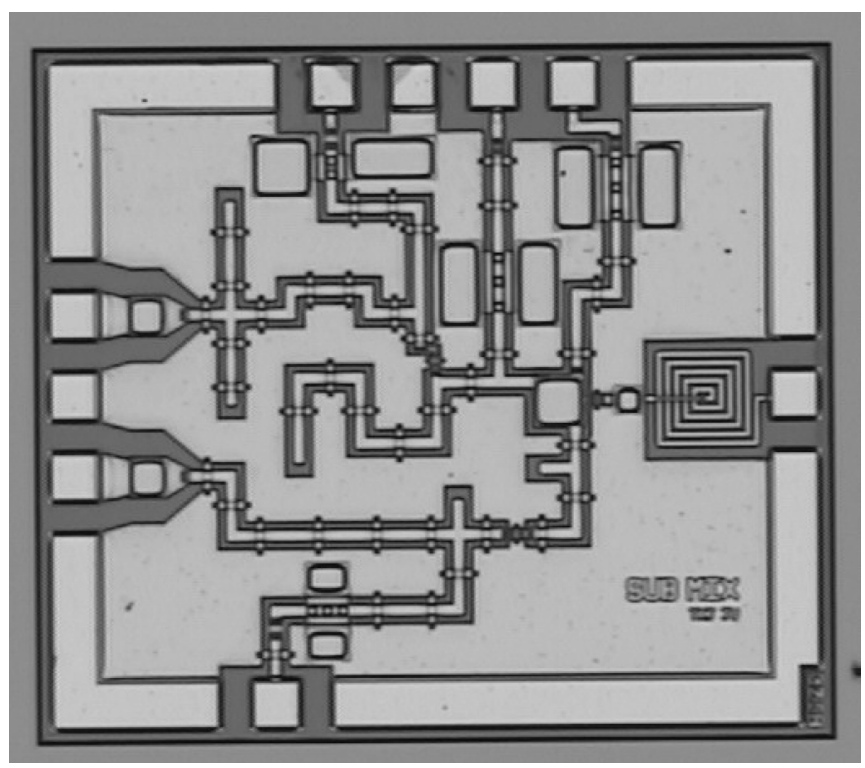

Fig. 6. Microphotograph of the SHM $\left(1.4 \times 1.6 \mathrm{~mm}^{2}\right.$ with pads $)$.

Ansoft's High Frequency Structure Simulator (HFSS) ${ }^{2}$ and ADS momentum, ${ }^{3}$ respectively.

The SHM was measured with on-wafer probes and the frequency-dependent cable and connector losses were carefully deembedded from the measurement results. The power consumption is $158 \mathrm{~mW}$ when biased from a single $+2.5-\mathrm{V}$ supply.

The conversion gain of the SHM versus the LO power is illustrated in Fig. 7 for an RF frequency of $f_{\mathrm{RF}}=50 \mathrm{GHz}$ with a power of $-27 \mathrm{dBm}$, which assures linear operation of the mixer at the RF port. The $\mathrm{LO}$ frequency is $f_{\mathrm{LO}}=26.25 \mathrm{GHz}$, which results in an IF frequency of $f_{\mathrm{IF}}=2 f_{\mathrm{LO}}-f_{\mathrm{RF}}=2.5 \mathrm{GHz}$. The measurements demonstrate typical conversion gain characteristic as a function of the LO power with a clear saturation region, which starts already at around $1 \mathrm{~mW}$. The agreement between simulations and measurements is very good, especially at high LO power levels, which are crucial for good mixer operation. At sufficient LO power level, the SHM presented here exhibits a gain of $G_{\text {conv }}>8 \mathrm{~dB}$. It should be emphasized that this gain is achieved without an IF amplifier, which could boost the gain even further. It should be further emphasized that this gain is achieved at relatively low LO power levels. Assuming a typical gain of an LNA in front of the mixer of $G_{\mathrm{LNA}} \approx 20 \mathrm{~dB}$, a receiver front-end using our SHM would exhibit a total gain of $>28 \mathrm{~dB}$ requiring only 20-40-dB gain on the IF side, which makes the design of the IF circuitry much easier.

The conversion gain achieved with our SHM is available over a wide RF frequency range of more than $10 \mathrm{GHz}$ with a maximum conversion gain of $10.3 \mathrm{~dB}$ around $45 \mathrm{GHz}$. The SHM can be operated over even wider bands, but then with a compromise in conversion gain, as indicated in Fig. 8. Fig. 8 presents the variation of the conversion gain versus the RF frequency, while keeping the IF frequency constant at $2.5 \mathrm{GHz}$. In the range between $40-50 \mathrm{GHz}$, the conversion gain is nearly constant with a small variation of around $\pm 1.5 \mathrm{~dB}$ over the frequency range. At

\footnotetext{
${ }^{2}$ HFSS v.9, Ansoft Corporation, San Jose, CA
}

${ }^{3}$ ADS 2004A, Agilent Technol Inc., Palo Alto, CA 


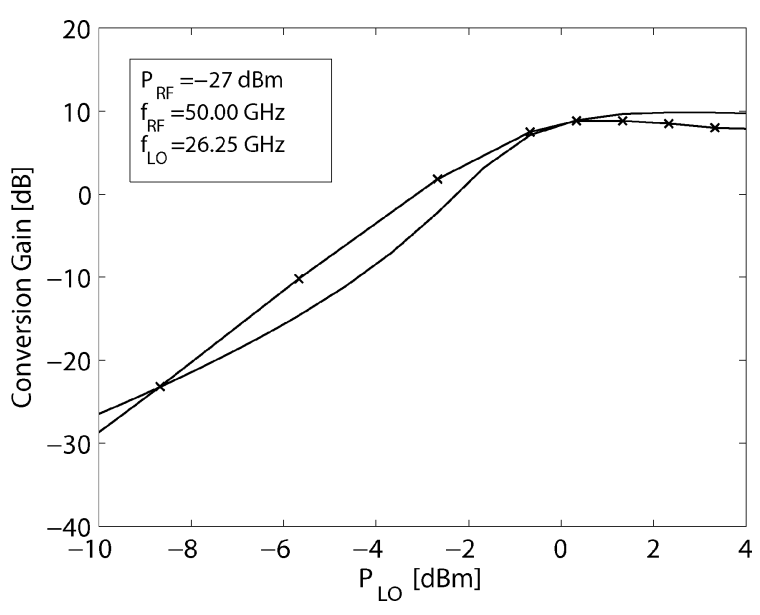

Fig. 7. Measured (symbols) and simulated (solid line) conversion gain versus LO power.

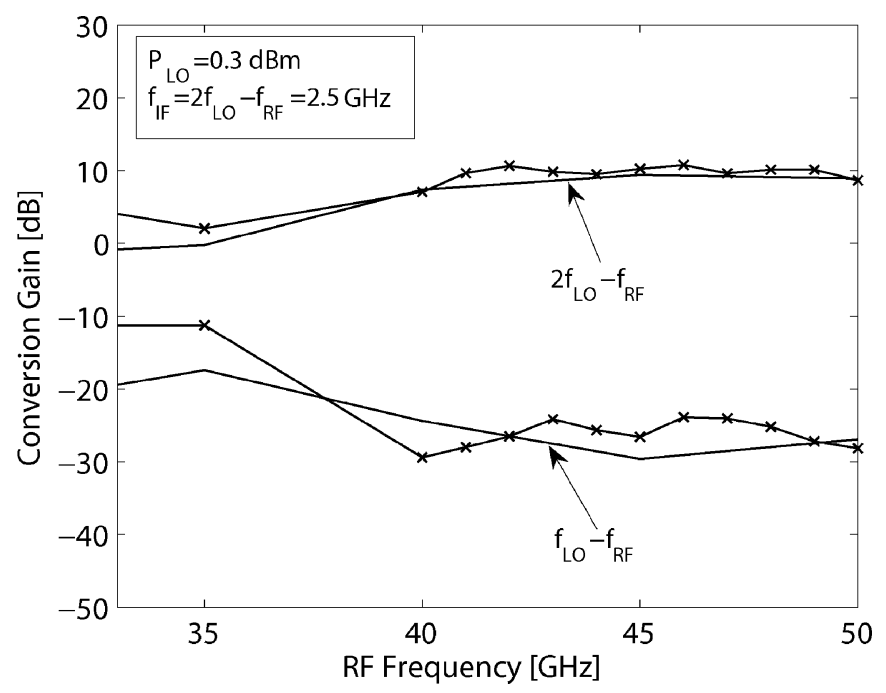

Fig. 8. Measured (symbols) and simulated (solid line) $Q$-band conversion gain for the subharmonic $2 f_{\mathrm{LO}}-f_{\mathrm{RF}}$ and fundamental $f_{\mathrm{LO}}-f_{\mathrm{RF}}$ mixing product. The IF for the SHM is $f_{\mathrm{IF}}=2.5 \mathrm{GHz}$.

lower frequencies, the conversion gain performance is limited mainly by the performance of the doubler circuit and by considerable mismatch in the combiner circuitry and the gain drops to values $G_{\mathrm{conv}} \gtrsim 1 \mathrm{~dB}$. The parasitic mixer output signal at $f_{\mathrm{IF}}=f_{\mathrm{LO}}-f_{\mathrm{RF}}$ is also included in the figure and is at least $24 \mathrm{~dB}$ below the desired signal at IF output. This is regarded as a good number for a single-ended mixer configuration.

The large-signal performance of the SHM has been evaluated using the gain compression characteristics of the mixer circuit. In Fig. 9, a comparison between measured and simulated output power at IF frequency versus the input power at the RF port. An excellent agreement between measurements and simulations is achieved up to power saturation. The SHM presented here exhibits an output referred 1-dB power compression point of $P_{\mathrm{IF}, 1 \mathrm{~dB}} \approx-6 \mathrm{dBm}$. The good agreement between measurements and simulations up to power saturation also proves the accuracy of the large-signal model employed in the design of the mixer. At very large powers, the SHM circuit seems to

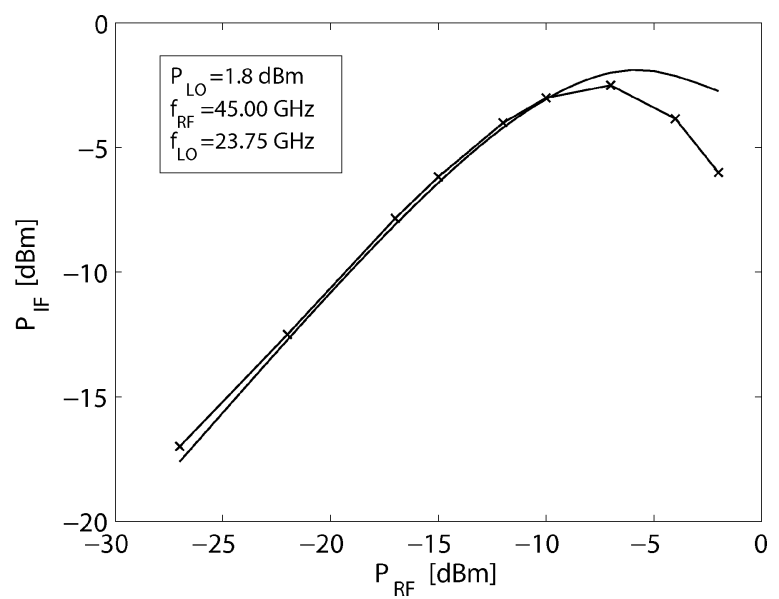

Fig. 9. Measured (symbols) and simulated (solid line) IF output power versus RF power.

saturate much faster as compared to the simulations; however, the trend is evaluated correctly.

Overall, the measured performance for the SHM is very well predicted by simulations. The good agreement is a result of accurate large-signal modeling of the InP DHBT devices, as well as EM simulation of passive structures.

A comparison of the results presented here and those published earlier points out the capabilities of the design and the technology employed in this paper. Such a comparison between different millimeter-wave SHMs is given in Table II. Previously reported SHM designs have mainly employed pHEMTs or Schottky barrier diodes in GaAs. More recently, SHM designs based on SiGe HBT devices have been reported with good millimeter-wave properties. To the authors' knowledge, our study represents the first reported SHM using InP DHBT devices. Many SHM published earlier integrate an IF boost stage with high gain to increase the signal level on the IF side. This is important in order to alleviate the necessity for an amplifier in the IF circuitry. We have demonstrated above that our SHM outperforms the SHM circuits presented in Table II with regard to critical parameters such as LO power, conversion gain, and output power. In order to make a fair comparison between various devices, the conversion gain without the IF buffer stage is reported in Table II. The estimated conversion gain of our SHM without the RF preamplifier stage is also stated in Table II. Even without the gain of the RF preamplifier, our SHM circuit is expected to provide a high conversion gain of $5.3 \mathrm{~dB}$. It should be noticed that the GaAs pHEMT SHM circuits in Table II also include the gain of an RF preamplifier stage. The operational frequency of our SHM is comparable to that of other GaAs pHEMT circuits, while most of the GaAs Schottky diode and the SiGe HBT-based SHMs operate at higher frequencies. The conversion gain of our SHM is almost double that of the best reported SHM circuits provided in Table II. It is interesting to note that at the same time the $1-\mathrm{dB}$ compression point, $P_{1 \mathrm{~dB}}$ of $-6 \mathrm{dBm}$, is comparable to that of the antiparallel Schottky barrier diode SHM reported in [14], which shows that the higher gain achieved in this paper is not at an expense of the power capabilities. It is further observed that our InP DHBT SHM design achieves the highest conversion 
TABLE II

MiLLIMETER-WAVE SHM PERFORMANCE

\begin{tabular}{|c|c|c|c|c|c|c|}
\hline Device & $f_{R F}[\mathrm{GHz}]$ & LO harm. & C.G. $[\mathrm{dB}]$ & $P_{1 d B}[\mathrm{dBm}]$ & $P_{L O}[\mathrm{dBm}]$ & Ref \\
\hline InP DHBT & $40-50$ & $2^{\text {nd }}$ & $>7\left(5.3^{+}\right)$ & -6.0 & 0.3 & This work \\
\hline GaAs pHEMT & 40 & $2^{\text {nd }}$ & 4.8 & -14.0 & 10.0 & {$[5]$} \\
\hline GaAs pHEMT & 60.4 & $4^{\text {th }}$ & 3.4 & -9.0 & 13.0 & {$[13]$} \\
\hline SiGe HBT & 122 & $2^{\text {nd }}$ & $4.0^{*}$ & NA & 3.0 & {$[3]$} \\
\hline SiGe HBT & 77 & $2^{\text {nd }}$ & $-10.3^{*}$ & -8.0 & 10.0 & {$[4]$} \\
\hline GaAs Schottky Diode & 94 & $2^{\text {nd }}$ & -7.0 & NA & 8.5 & {$[2]$} \\
\hline GaAs Schottky Diode & 94 & $2^{\text {nd }} / 4^{t h}$ & $-9.2 /-11.4$ & -6.0 & $5.0 / 10.0$ & {$[14]$} \\
\hline GaAs Schottky Diode & 44 & $2^{\text {nd }}$ & -8.0 & -2.0 & 9.0 & {$[15]$} \\
\hline
\end{tabular}

$\left({ }^{+}\right.$Conversion gain w/o RF preamplifier. ${ }^{*}$ Conversion gain w/o IF output buffer. )

gain over a wide bandwidth with the lowest LO input power. Our circuit operates at LO power levels at least a factor of 10 lower than comparable SHM circuits. The noise figure and intermodulation properties of the mixer could not be measured due to lack of suitable millimeter-wave test equipment.

\section{CONCLUSION}

This paper reported the design, fabrication, and characterization of a $Q$-band SHM using InP DHBT devices. It is believed to be the first demonstration of an SHM using InP DHBT MMIC technology. We have presented a design procedure, which greatly facilitates the design of the SHM circuit, which can accurately predict the required LO power level, but is too optimistic with regard to conversion gain predictions, due to its simplicity. The design procedure is centered around an analytical formulation of the conversion gain in the SHM, which provides not only the LO power level, but also the bias conditions for the transistors and the matching for the respective devices.

An accurate design of the SHM requires a subsequent largesignal simulation and an accurate determination of the passive circuit structure, which is obtained from full-wave EM simulations. The large-signal harmonic balance simulations are employing an accurate large-signal model presented earlier. This is believed to be crucial for the good agreement between predicted and measured performance of the SHM circuit presented here.

The performance of the SHM is believed to be better with regard to conversion gain and LO power level as compared to other earlier published SHM. The circuit exhibits a conversion gain of $>7 \mathrm{~dB}$ when operated with an LO power level of only $1 \mathrm{~mW}$. The circuit can deliver an output power around $0.25 \mathrm{~mW}$ at the IF frequency before conversion gain compression. This performance has been measured across an RF frequency band of $40-50 \mathrm{GHz}$ and it is believed that this performance can also be achieved at higher frequencies with frequency scaled designs.

\section{REFERENCES}

[1] S. A. Maas, Microwave Mixers, 2nd ed. Norwood, MA: Artech House, 1993.

[2] S. Raman et al., "A high-performance $W$-band uniplanar subharmonic mixer," IEEE Trans. Microw. Theory Tech., vol. 45, no. 6, pp. 955-962, Jun. 1997.

[3] A. Müller et al., "A $122 \mathrm{GHz}$ SiGe active subharmonic mixer," in Proc. 13th GAAS Symp., Paris, France, Oct. 2005, pp. 57-60.

[4] J. Hung et al., "A $77 \mathrm{GHz}$ SiGe sub-harmonic balanced mixer," IEEE J. Solid-State Circuits, vol. 40, no. 11, pp. 2167-2173, Nov. 2005.
[5] B. H. Lee et al., " $Q$-band high conversion gain active sub-harmonic mixer," Current Appl. Phys., no. 4, pp. 69-73, 2004.

[6] T.-Y. Yang and H.-K. Chiou, "A $28 \mathrm{GHz}$ sub-harmonic mixer using LO doubler in $0.18 \mu \mathrm{m}$ CMOS technology," in IEEE RFIC Symp. Dig., Jun. 2006, pp. 209-212.

[7] S. A. Maas, Nonlinear Microwave and RF Circuits, 2nd ed. Norwood, MA: Artech House, 2003.

[8] T. K. Johansen et al., "A novel HBT frequency doubler design for millimeter-wave applications," in Proc. INMMIC, Jan. 2006, pp. 106-109.

[9] G. Boeck, K. Schmidt, and M. Tempel, "Planar microwave mixers for wireless and sensor applications," in Proc. SBMO/IEEE MTT-S IMOC 2001, 2001, pp. 233-243.

[10] T. K. Johansen et al., "Large-signal modeling of high-speed InP DHBTs using electromagnetic simulation based de-embedding," in IEEE MTT-S Int. Microw. Symp. Dig., Jun. 2006, pp. 655-658.

[11] S. P. O. Bruce et al., "Design and realization of a millimeter-wave $\mathrm{Si} / \mathrm{SiGe} \mathrm{HBT}$ frequency multiplier," IEEE Trans. Microw. Theory Tech., vol. 46, no. 5, pp. 695-700, May 1998.

[12] S. Blayac et al., "MSI InP/InGaAs DHBT technology: Beyond 40 Gbit/s circuits," in 14th Indium Phosphide and Rel. Mat. Conf., 2002, pp. 51-54.

[13] S. C. Kim et al., "High conversion gain cascode quadruple subharmonic mixer for millimeter-wave applications," Current Appl. Phys., no. 5, pp. 231-236, 2005.

[14] K. Kanaya et al., "A $94 \mathrm{GHz}$ high performance quadruple subharmonic mixer MMIC," in IEEE MTT-S. Int. Microw. Symp. Dig., Jun. 2002, pp. $1249-1252$.

[15] K. Hettak et al., "A novel uniplanar $44 \mathrm{GHz}$ MMIC subharmonic mixer using CWP series stubs," in IEEE MTT-S Int. Microw. Symp. Dig., Jun. 2004, pp. 1157-1160.

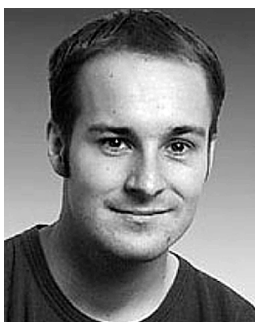

Tom K. Johansen (S'02-M'04) received the M.S. and Ph.D. degrees in electrical engineering from the Technical University of Denmark, Denmark, in 1999 and 2003, respectively.

In 1999, he joined the Electromagnetic Systems Section, Oersted • DTU Department, Technical University of Denmark, where he is currently an Associate Professor. From September 2001 to March 2002, he was a Visiting Scholar with the Center for Wireless Communication, University of San Diego, San Diego, CA. His research areas include the modeling of HBT devices, nonlinear circuit analysis, millimeter-wave integrated circuit design, and EM simulation.

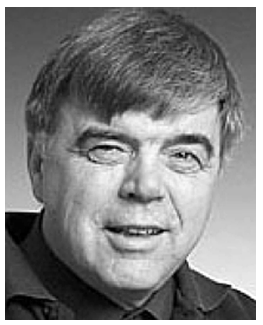

Jens Vidkjær (S'72-M'72) received the M.S. and Ph.D. degrees from the Technical University of Denmark, Lyngby, Denmark, in 1968 and 1975, respectively.

Since 1970, he has been with the Electronics Laboratory and the Semiconductor Laboratory, Technical University of Denmark, where he is currently a Reader with the Electromagnetic System Group, Oersted Institute. His research areas have covered RF-power amplifier design, computer-aided design (CAD) methods, device modeling, measurement accuracies, and MMIC design. 


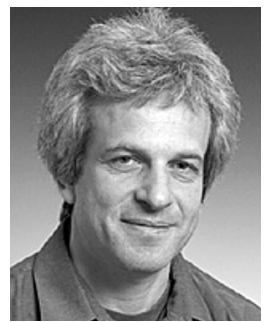

Viktor Krozer (M'91-SM'03) received the Dipl.-Ing. and Dr.-Ing. degrees in electrical engineering from the Technical University of Darmstadt (TU Darmstadt), Darmstadt, Germany, in 1984 and 1991, respectively.

In 1991, he became a Senior Scientist with TU Darmstadt, where he was involved with high-temperature microwave devices and circuits and submillimeter-wave electronics. From 1996 to 2002, he was a Professor with the Technical University of Chemnitz, Chemnitz, Germany. Since 2002, he has been a Professor with the ElectroScience Section, Ørsted-DTU Department, Technical University of Denmark, Lyngby, Denmark, where he heads the Microwave Technology Group. His research areas include terahertz electronics, MMICs, nonlinear circuit analysis and design, device modeling, and remote sensing instrumentation.

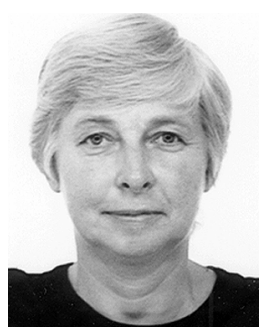

Agnieszka Konczykowska (M'88-SM'97) received the M.S. degree in applied mathematics and Ph.D. degree in electrical engineering from the Warsaw University of Technology, Warsaw, Poland, in 1971 and 1977, respectively.

From 1971 to 1982 , she was with the Warsaw University of Technology. From 1982 to 2001, she was with the France Telecon Research Center. Since 2001, she has been the Leader of the High Speed Circuits Group, Alcatel-Thales III-V Laboratory, Marcoussis, France. She has been involved in different domains of computer-aided design (CAD) and design methodologies such as methods and applications of symbolic analysis, semiconductor device modeling, and analysis and design of switched-capacitor circuits. She is a member of the Editorial Board of the International Journal of Circuit Theory and Applications. Her current research interests are linked with the circuit design of very high-speed digital circuits for optical communications in InP HBT technology. She has coauthored over 180 scientific publications.

Dr. Konczykowska has been a member of many technical committees of international conferences. She is a reviewer for numerous technical journals. She was the president of the European Circuit Society (1995-1999).

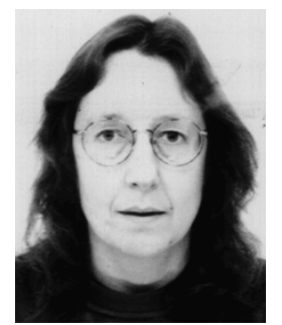

Muriel Riet was born in Choisy-le-Roi, France, in 1958. She received the Ph.D. degree in electronic engineering from the University of Paris XI, Paris, France, in 1985.

In 1985, she joined CNET, Research Center of France Telecom, where she studied compound semiconductor HBTs. She is currently in charge of InP HBT technology for high bit-rate optical communications up to $40 \mathrm{~Gb} / \mathrm{s}$ with the Alcatel-Thales III-V Laboratory, Marcoussis, France.

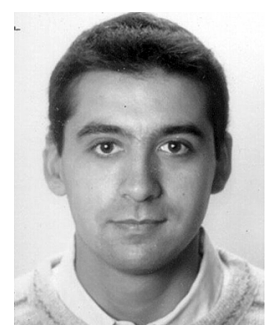

Filipe Jorge was born in Roubaix, France, in 1970. $\mathrm{He}$ received the Ph.D. degree in electronic engineering from the University of Lille 1, Villeneuve d'Ascq, France, in 1999.

In 2000, he joined OPTO+, an Alcatel Research and Innovation Unit, as a Research and Development Engineer. He is currently involved with module design and characterization of high-speed circuits for optical communication systems with the $\mathrm{Al}$ catel-Thales III-V Laboratory, Marcoussis, France.

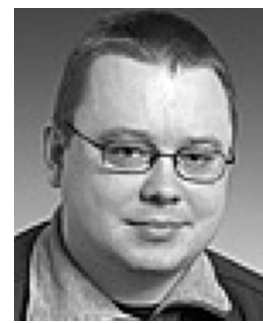

Torsten Djurhuus received the M.S. degree in electrical engineering from the Technical University of Denmark, Lyngby, Denmark, in 2003, and is currently working toward the Ph.D. degree at the Technical University of Denmark.

His research interests include nonlinear circuit analysis, MMIC design, and RF oscillator design. 